\title{
Impact of the different mutations in the cystic fibrosis gene in children with chronic rhinosinusitis
}

\begin{abstract}
Background: Cystic fibrosis (CF) is a worldwide disease occurring mostly in caucasians. It is an autosomal recessive disorder that leads to a malfunction of CF transmembrane conductance regulator (CFTR). These mutations cause an ionic disorder on the body fluids and a modification on its consistency. Affects multiple parts of the body and rhinosinusitis is a common manifestation of the upper airway affection.

Material and methods: This retrospective study performed a statistical analysis of the prevelence of chronic rhinosinusitis with polyposis, genotipe and mortality in 30 children under 18 years with cystic fibrosis followed in the CF unit of Coimbra University Hospital.

Results: The mean age of this study was 12,9 years. Phenylalanine deletion at position 508 (F508delF508del) was the most prevalent genotype $(66,7 \%)$. Females patients had an higher prevalence of morbidities, however male patients had an higher mortality rate $20 \%$ comparing to $6,7 \%$. Nasal polyposis was present only in the living ones with F508delF508del genotype. ENT (ear, nouse and throut) symptons and an abnormal ENT examination were mostly observed in F508delF508del genotype.

Conclusions: $\mathrm{CF}$ is a lifelong disease that requires long-term surveillance and compliance. The involvement of the lower airway is prevalent in young chlidren. The uper airway symptoms becomes more important with disease progression. Nasal poliposis is prevalent on the older ones with F508delF508del genotype. In this kind of patients with persistent symptoms, who have failed medical management, are often considered appropriate candidates for functional endoscopic surgery.
\end{abstract}

Keywords: chronic rhinosinusitis, cystic fibrosis, nasal polyposis, children
Volume II Issue 5 - 2019

\author{
Mafalda da Silva Ferreira,' João Elói Moura,' \\ Ana Margarida Amorim,' Ana Bernardo \\ Ferreira, ${ }^{2}$ Luís Filipe Silva' \\ 'ENT Department, Centro Hospitalar e Universitário de \\ Coimbra, Portugal \\ 2Pediatric Department, Centro Hospitalar entre Douro e Vouga, \\ Portugal
}

Correspondence: Mafalda Silva Ferreira, ENT Department, Coimbra University Hospital, Rua Professor Mota Pinto 3000075, Coimbra, Portugal, Tel 239400400,

Email Mafalda.sf1990@gmail.com

Received:September 04, 2019 | Published: September 10,

2019 Abbreviations: CF, cystic fibrosis; CFTR, CF transmembrane
conductance regulator

\section{Introduction}

Decades ago cystic fibrosis had a high rate of early mortality making it a frightening disease among the medical community. This high rate was related to the pulmonar deterioration characteristic of this pathology and due to opportunistic bactéria. ${ }^{1,2}$ Advances in knowledge of CF physiopathology, improvement in therapeutics and vaccines prometed an increase in patients survival and quality of life which also led to the emergence of new comorbidities. ${ }^{3,4}$ $\mathrm{CF}$ is more prevalent in Caucasians and is an autosomal recessive disorder genetically inherited, caused by some particular dysfunction or deficiency of the $\mathrm{CF}$ transmembrane conductance regulator. ${ }^{5-7}$ CFTR gene is located on the long arm of chromosome 7 and the commonest mutation is the deletion of phenylalanine at codon 508 . CFTR is an apical membrane anion channel, that can be found in multiple locals like the respiratory and exocrine glandular epithelium. It regulates liquid volume on epithelial surfaces through chloride secretion and inhibition of sodium absorption. ${ }^{8}$ The absence of this chloride channel leads to an imbalance of ion concentrations across the cell membrane and this results a more viscous fluid secreted by the glands and posterior atrophy of glands ducts. ${ }^{9}$ It also leads to a failure of mucociliary clearance, a disability to effectively clear inhaled bacteria, and in turn an excessive inflammatory response to pathogens. Cystic fibrosis used to be a digestive and lung disease of younger ages but more recently has become a complex and multisystem disease. At upper airway level the abnormal ionic and water fluxes alter the viscoelastic properties of mucins, leading to fluid hyperviscosity and mucociliary dysfunction. This new state leads to a paranasal sinus drainage obstruction which predisposes to the development of local inflammation and bacterial colonization particularly by Pseudomonas aeruginosa and Staphylococcus aureus. ${ }^{10}$ The involvement of the upper airways, mainly due to pathological alterations of the paranasal sinus is common in patients with $\mathrm{CF}$, although many have few symptoms. ${ }^{11}$

\section{Material and methods}

A total of 30 patients and their medical records, followed in CF unit at Coimbra University Hospital, were retrospectively analyzed. It was made an overall characterization of the population, collected genotype, symptoms and treatment. Statistical analysis was performed using IBM SPSS version 25 with statistical significance assumed at $\mathrm{p}<0.05$. Chi-square and Fisher's Exact tests were used to determine group differences in demographic and clinical variables. Univariate analysis was performed to outline predictive factors for mortality. Experienced otorhinolaryngologists reviewed the data.

\section{Results}

Of 30 patients enrolled in this study, 15 patients were male and 15 were female with a mean age of 12,9 years. Phenylalanine deletion at position 508 (F508delF508del) was the most prevalent genotype (66,7\%), while F508del7111GT and F508delc.3321dup were the 
least common (3,3\%). Females patients had an higher prevalence of morbidities, such as fatigue $(71,4 \%)$ and weight loss $(57,1 \%)$. The mortality rate for male patients was $20 \%$ (ages between 15 and 17 years old), comparing to $6,7 \%$ in female patients (17 years old). However, no statistically significant differences between genders regarding genotypes, nasal polyposis, morbidities, and mortality were found. Table 1 compares the study population between genders. Influence of cystic fibrosis genotype on symptoms and physical examination is shown in Table 2. Less frequent genotypes (F508del2184insA, N1303KA561E, F508delG542x, F508del7111GT, F508delc.3321dup, F508delR334w and F508del3171delC mutations) were grouped for

Table I Clinical features of CF patients per gender statistical purposes. Considering respiratory symptoms, both groups had high prevalence of sputum and cough. However, ENT (Ear, Nose, and Throat) symptoms, like nasal obstruction and rhinorrhea were only detected in a patient with the F508delF508del genotype (Figure 1). The presence of ENT symptoms did not correlate with an abnormal ENT physical examination. In fact, an abnormal ENT examination was only present in F508delF508del genotypes. Chisquare and Fisher's Exact tests were used to assess dependence between genotype and symptoms and physical examination, but no statistically significant differences were found.

\begin{tabular}{|c|c|c|c|c|}
\hline \multirow{2}{*}{ Genotype,\% } & \multirow{2}{*}{$\begin{array}{l}\text { Frequency } \\
(n=30)\end{array}$} & \multicolumn{2}{|l|}{ Gender } & \multirow{2}{*}{$p$ value* } \\
\hline & & Male $(n=\mid 5)$ & Female $(n=15)$ & \\
\hline F508del2 I84insA & $6,7 \%$ & $6,7 \%$ & $6,7 \%$ & \\
\hline NI303KA56IE & $6,7 \%$ & $13,3 \%$ & $0,0 \%$ & \\
\hline F508delG542x & $6,7 \%$ & $6,7 \%$ & $6,7 \%$ & \\
\hline F508del7IIIGT & $3,3 \%$ & $0,0 \%$ & $6,7 \%$ & .851 \\
\hline F508delF508del & $66,7 \%$ & $60,0 \%$ & $73,3 \%$ & \\
\hline F508delc.332 I dup & $3,3 \%$ & $6,7 \%$ & $0,0 \%$ & \\
\hline F508delR334w & $6,7 \%$ & $6,7 \%$ & $6,7 \%$ & \\
\hline Nasal polyposis, \% & & $6,7 \%$ & $20,0 \%$ & .598 \\
\hline \multicolumn{5}{|l|}{ Comorbidities, \% } \\
\hline Fatigue & & $28,6 \%$ & $71,4 \%$ & .390 \\
\hline Weight loss & & $42,9 \%$ & $57,1 \%$ & 1.000 \\
\hline Deceased, \% & & $20,0 \%$ & $6,7 \%$ & .598 \\
\hline
\end{tabular}

*p-value was calculated using Chi-square and Fisher's Exact Tests.

Table 2 Clinical features of CF patients per genotype

\begin{tabular}{llll}
\hline & F508delF508del & Other genotypes* & p value** \\
\hline Abnormal ENT examination, $\%$ & $35,0 \%$ & $0,0 \%$ & .064 \\
Nasal polyposis, $\%$ & $20,0 \%$ & $0,0 \%$ & .272 \\
\hline Respiratory symptoms, \% & & & .380 \\
\hline Sputum & $73,7 \%$ & $77,8 \%$ & .449 \\
Cough & $78,9 \%$ & $66,7 \%$ & .195 \\
Wheeze & $5,3 \%$ & $11,1 \%$ & .735 \\
\hline ENT symptoms, $\%$ & & $0,0 \%$ & 1.000 \\
\hline Nasal obstruction & $42,1 \%$ & $0,0 \%$ & .584 \\
Rhinorrhea & $26,3 \%$ & $20,0 \%$ & \\
\hline Deceased, \% & $10,0 \%$ & &. \\
\hline
\end{tabular}

*Other genotypes include F508del2 I84insA, NI303KA56 IE, F508delG542x, F508del7 I I IGT,

F508delc.332 I dup, F508delR334w and F508del3I7I delC mutations.

**p value was calculated using Chi-square and Fisher's Exact tests. 


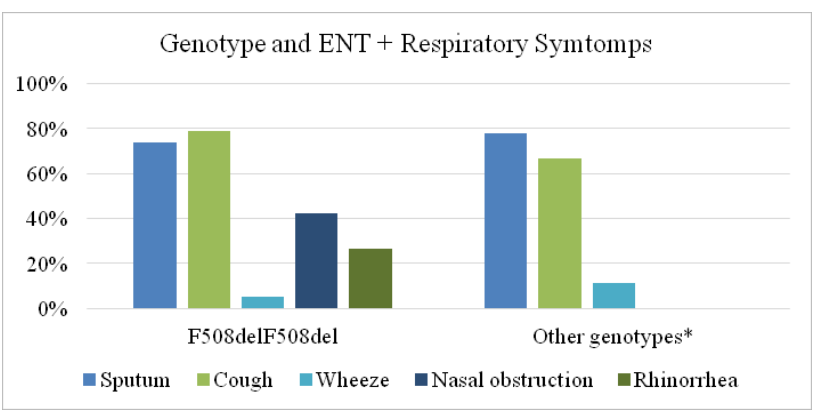

Figure I Genotype and ENT + Respiratory symptoms.

Nasal polyposis was present only in currently living patients. Half of these patients went to surgery (nasal endoscopic surgery) and all of them had polyposis recurrency. The other half perform topical steroids and surveillance. Chi-square test shows a significant association between mortality and the presence of previous fatigue $(\mathrm{p}=.031)$. The prevalence of previous fatigue concomitant with Cystic Fibrosis showed to be statistically higher in deceased patients comparing to the currently living ones. Genotype did not show to be a predictive factor for mortality.

\section{Discussion}

CF is caused by a malfuction of CFTR. The most common mutation is the deletion of phenylalanine ate codon 508 accounting for about $70 \%$, but more than 850 mutant alleles have been reported. Different mutations in this gene have multiple effects on CFTR function and can result in different phenotypes of the disease. ${ }^{12}$ As observed in our study the most prevalente genotype was F508delF508del, however six other were found. The nasal phenotype (upper airway symptoms) was only detected in patients with F508delF508del genotype. This finding restricts children population which will futurly concern about nasosinusal pathologies. Cystic fibrosis used to be a digestive and lung disease of younger ages but more recently has become a more complex and multisystemic disease. This changes are consequence of the treatment improvement, increase of vaccination plan and an early CF screening and diagnosis which in turn leads to an increased survival. The predicted median survival for babies born in the $21 \mathrm{st}$ century is now more than 40 years. ${ }^{13}$ In our work we could conclude that females children had an higher prevalence of morbidities, such as fatigue and weight loss, and therefore have a longer and painful symptomatic periods. This is an important fact during the approach of CF patients, since we must be more cautious with female complaints. Nevertheless, the mortality rate for male patients was $20 \%$, comparing to $6,7 \%$ in female patients. Male gender is therefore more susceptible to decease from this disease in spite of manifesting few incapacitating symptoms. The prevalence of previous fatigue in CF patients showed to be statistically higher in deceased patients. The presence of fatigue in CF children complaints is a bad prognostic factor, and must be approach promptly and cautiously.

The lining of the sinonasal epithelium of airway surface contains a low viscosity periciliary fluid layer and a superficial mucus layer, which trap inhaled particles and through a coordinated mucociliary clearance leads them into the digestive tract. Intact mucociliary clearance is considered the airway's innate defense against diseases. ${ }^{3}$ Mucociliary clearance is grossly affected by the disturbance in anion transportation due to CFTR mutation. This fact increase mucus viscosity and consequent obstruction of sinus ostia. It creates an hypoxic condition with increased edema, secondary ciliary dyskinesia, and subsequent bacterial overgrowth in CF patients. This chronic inflammatory state promotes an increase of local neutrophils and finally a formation of neutrophil-predominant nasal polyps..$^{14,15}$

Nasal polyposis was present only in the living children of our study. We can suppose that in some time, during the course of the disease, it must occur a proper inflammatory condition for polyposis development. So it is expected to find chronic rhinosinusitis in the older children. According to another studies younger children present mostly lower airway symptoms and morbidities. Sputum and cough were the most frequent symptoms in this study due to our younger population. Older ones and adults present more upper airway symptoms and commitment. ${ }^{8}$ The lower airway commitment is more incapacitating and critical than the upper airway conditions. Mortality was observed in early ages; however our mortality rates were low by the effort of a permanent surveilliance and accurate treatment.

Half of the patients with nasal polyposis perform surgery (functional endoscopic sinus surgery) but all of them recurred. The other half performs topical steroids. We first attempt to control ENT symptoms and nasal polyposis with medication but when conservative treatment does not resolve the symptoms and sinonasal disease is related to deterioration of lung disease surgical intervention is performed. Yet, recurrence rates and clinical deterioration remains high, as observed in our study. Surgery aims to give the patient better quality of life by reducing nasal symptoms. ${ }^{16}$ However, the diseased mucosa remains after surgery as so the disturbance to clear the viscous mucus. Due to this altered mucus and chronic bacterial infection the growth of granulation tissue is stimulated, leading to the clinical appearing of recurrent polyposis. ${ }^{17}$

\section{Conclusions}

$\mathrm{CF}$ continues to be a life-threatening inherited disease. Chronic rhinosinusitis with nasal polyposis is much more frequent in older ages. The pathogenesis of nasal polyposis is characterized by impaired mucociliary clearance, resulting in bacterial colonization and neutrophil-dominated host reaction. It can present itself with a few symptoms for the patient and a low morbidity. However, severe upper airway symptoms may be treated aggressively. Clinical practice and treatment has significantly improved. Therefore, clinical morbidities and mortality rates have been decreasing with the opposite increase of life expectancy. Much less lower airways conditions will be observed in our CF population. Instead upper airways affections will appear. That is why we must improve our knowledge about this particular disease and bring up new therapeutic approaches.

\section{Acknowledgments}

None.

\section{Conflict of interests}

The authors declare that there is no conflict of interests regarding the publication of this paper.

\section{Funding details}

None. 


\section{References}

1. Massie J, Robinson PJ, Cooper PJ. The story of cystic fibrosis 19652015. J Paediatr Child Health. 2016;52(11):991-994.

2. Kocha C, Høibyb N. Diagnosis and Treatment of Cystic Fibrosis. Respiration. 2000;67(3):239-247.

3. Haaban M, Kejner A, Rowe S, et al. Cystic Fibrosis Chronic Rhinosinusitis: A Comprehensive Review. Am J Rhinol Allergy. 2013;27(5):387-395.

4. Farrell P, White T, Ren CL, et al. Diagnosis of Cystic Fibrosis: Consensus Guidelines from the Cystic Fibrosis Foundation. J Pediatr. 2017;181:S4-S15.

5. Cutting G. Cystic fibrosis genetics: from molecular understanding to clinical application. Nat Rev Genet. 2014;16(1):45-56.

6. Karen ZV, Clement LR. Diagnosis of Cystic Fibrosis. Clinic Rev Allerg Immunol. 2008;35:100-106.

7. Khatami GR, Mir-Nasseri MM, Seyghali F, et al. Characteristics of Patients with Cystic Fibrosis: Experience in a Large Referral Children's Hospital in Tehran, Iran. Middle East J Dig Dis. 2010;2(1):20-23.

8. Davies JC, Alton EWFW, Bush A. Cystic fibrosis. The BMJ. 2007;335(7632):1255-1259.

9. Vankeerberghen A, Cuppens H, Cassiman J. The cystic fibrosis transmembrane conductance regulator: an intriguing protein with pleiotropic functions. J Cyst Fibros. 2002;1(1):13-29.
10. Cunha A, Amorim A, Augusto S, et al. Fibrose quística em adultos Experiência do Serviço de Otorrinolaringologia do Centro Hospitalar e Universitário de Coimbra. Acta Otorrinolaringológica Gallega. 2016;9(1):79-88.

11. Le C, McCrary HC, Chang E. Cystic Fibrosis Sinusitis. Adv Otorhinolaryngol. 2016;79:29-37.

12. Zielenski J. Genotype and Phenotype in Cystic Fibrosis. Respiration. 2000;67(2):117-133.

13. Proesmans M. Best practices in the treatment of early cystic fibrosis lung disease. Ther Adv Respir Dis. 2017;11(2):97-104.

14. Karanth T, Karanth V, Ward B, et al. Medical interventions for chronic rhinosinusitis in cystic fibrosis. Cochrane Database of Systematic Reviews. 2018.

15. Hamilos DL. Chronic Rhinosinusitis in Patients with Cystic Fibrosis. $J$ Allergy Clin Immunol Pract. 2016;4(4):605-612.

16. Mainz JG, Koitschev A. Pathogenesis and Management of Nasal Polyposis in Cystic Fibrosis. Curr Allergy Asthma Rep. 2012; 12(123):163-174.

17. Kang SH, Dalcin PTR, Piltcher OB, et al. Chronic rhinosinusitis and nasal polyposis in cystic fibrosis: update on diagnosis and treatment. $J$ Bras Pneumol. 2015;41(1):65-76. 